\title{
Managing fatigue after cancer treatment: development of RESTORE, a web-based resource to support self- management
}

\author{
C. Foster'*, L. Calman', C. Grimmett', M. Breckons', P. Cotterell', L. Yardley², J. Joseph², S. Hughes², R. Jones², \\ C. Leonidou', J. Armes ${ }^{3}$, L. Batehup', J. Corner', D. Fenlon', E. Lennan', C. Morris', A. Neylon 4, E. Ream5, L. Turner' \\ and A. Richardson' \\ 'Faculty of Health Sciences, University of Southampton, Southampton, SO I 7 IBJ, UK \\ ${ }^{2}$ School of Psychology, University of Southampton, Southampton, SOI7 IBJ, UK \\ ${ }^{3}$ Florence Nightingale School of Nursing and Midwifery, Kings College London, London, SWI 8WA, UK \\ ${ }^{4}$ Macmillan Cancer Support, London, SEI 7UQ, UK \\ ${ }^{5}$ School of Health Sciences, University of Surrey, Guildford, GU2 7TE, UK
}

*Correspondence to:

Faculty of Health Sciences,

University of Southampton,

Southampton, SO 17 IBJ, UK.

E-mail: c.l.foster@soton.ac.uk

Received: 16 July 2014

Revised: 3 December 2014

Accepted: 16 December 2014

\begin{abstract}
Objective: The aim of this study is to co-create an evidence-based and theoretically informed web-based intervention (RESTORE) designed to enhance self-efficacy to live with cancer-related fatigue (CRF) following primary cancer treatment.

Methods: A nine-step process informed the development of the intervention: (1) review of empirical literature; (2) review of existing patient resources; (3) establish theoretical framework; (4) establish design team with expertise in web-based interventions, CRF and people affected by cancer; (5) develop prototype intervention; (6) user testing phase 1; (7) refinement of prototype; (8) user testing phase 2; and (9) develop final intervention.

Results: Key stakeholders made a critical contribution at every step of intervention development, and user testing, which involved an iterative process and resulted in the final intervention. The RESTORE intervention has five sessions; sessions 1 and 2 include an introduction to CRF and goal setting. Sessions 3-5 can be tailored to user preference and are designed to cover areas of life where CRF may have an impact: home and work life, personal relationships and emotional adjustment.

Conclusions: It is feasible to systematically 'co-create' an evidence-based and theory-driven web-based self-management intervention to support cancer survivors living with the consequences of cancer and its treatment. This is the first account of the development of a web-based intervention to support self-efficacy to manage CRF. An exploratory trial to test the feasibility and acceptability of RESTORE is now warranted.

Copyright $@ 2015$ John Wiley \& Sons, Ltd.
\end{abstract}

\section{Background}

More than 25 million people worldwide are living with or beyond a cancer diagnosis [1]. Improvements in detection and treatments have contributed to rising survival rates with many people faring well after treatment. However, consequences of cancer and its treatment can have a considerable and long-term impact on everyday life [2,3]. With aftercare systems not meeting the needs of patients [4], an ageing population and stretched healthcare systems, there is growing concern about how best to support cancer survivors [5]. As a consequence, aftercare for cancer patients is changing.

One of the most prevalent and debilitating consequences of cancer and its treatment is cancer-related fatigue (CRF), a multifaceted symptom that can have a profound impact on all aspects of daily life and affects at least a third of people following primary treatment [6]. Our research indicates that this can be significantly higher in the year following primary treatment [7]. Fatigue is complex with physical (e.g. low energy or need to rest), cognitive (e.g. reduced concentration or attention) and affective (e.g. decreased motivation or interest) elements [8]. There is emerging evidence of the benefit of physical activity, cognitive behaviour therapy (CBT) and psychosocial support in the management of CRF [9]. Most published studies concentrate on patients during treatment and do not address the ongoing difficulties faced by people once cancer treatment has ended.

Hoffman and colleagues (2009) identified that perceived self-efficacy mediates the association between $\mathrm{CRF}$ and physical function [10]. Other data suggest selfefficacy to manage CRF impacts on CRF levels $[8,11]$ and may also mediate the relationship between physical 
activity and improved CRF [12]. Hoffman et al. therefore make the case for the development of interventions to enhance self-efficacy to self-manage CRF.

Self-management is becoming an essential part of tailored follow-up with care pathways adapted to an individual's needs [5]. People may want support in a variety of forms to manage the impact of cancer on their lives, but the cancer diagnosis and treatment can leave them feeling vulnerable and lacking in confidence, which may act as a barrier to accessing support [13]. Rebuilding confidence is an important part of recovery, and if patients can be supported to do this, they will be in a better position to self-manage problems and access support, which will influence whether individuals 'live well' after treatment [13]. The Internet can be an effective mode of delivery of health care for self-management of long-term conditions and behaviour change [14]. It is increasingly being used as a resource by cancer survivors [15]. Use of the Internet by people over 65 years old is steadily growing [16], and web-based interventions have been used successfully by older people [17].

This paper describes the development and early testing of a web-based intervention (RESTORE) designed to enhance people's confidence to live with CRF following primary cancer treatment. The development of the intervention was informed by the Medical Research Council's guidelines on developing complex interventions [18]. RESTORE was co-created with service users and academic, clinical and third-sector stakeholders. There has been a call for the publication of more detailed descriptions of development work that underpins complex interventions in order to build on previous literature and advance the field of behavioural medicine [19]. This paper provides a detailed description of each stage of development of RESTORE and presents an exemplar for developing and testing similar web-based interventions.

\section{Development}

There is widespread support that the development of interventions should be informed by psychological theory and evidence of effectiveness [18]. In the case of web-based interventions, the principles of web design and user testing are also important [20]. Each step of the development and user testing of RESTORE is illustrated in Figure 1. The intervention was co-created with a range of stakeholders. Initial ideas for the intervention, and user engagement in the study, were discussed as part of 'Knowledge Café' events held in 2009 and again in 2012. The aim of the 2009 event was to form a user reference group including people affected by cancer who were interested in the team's programme of research. Conceptual ideas for the programme were discussed, and attendees were encouraged to critically appraise plans, drawing on personal experiences and highlighting important domains that were

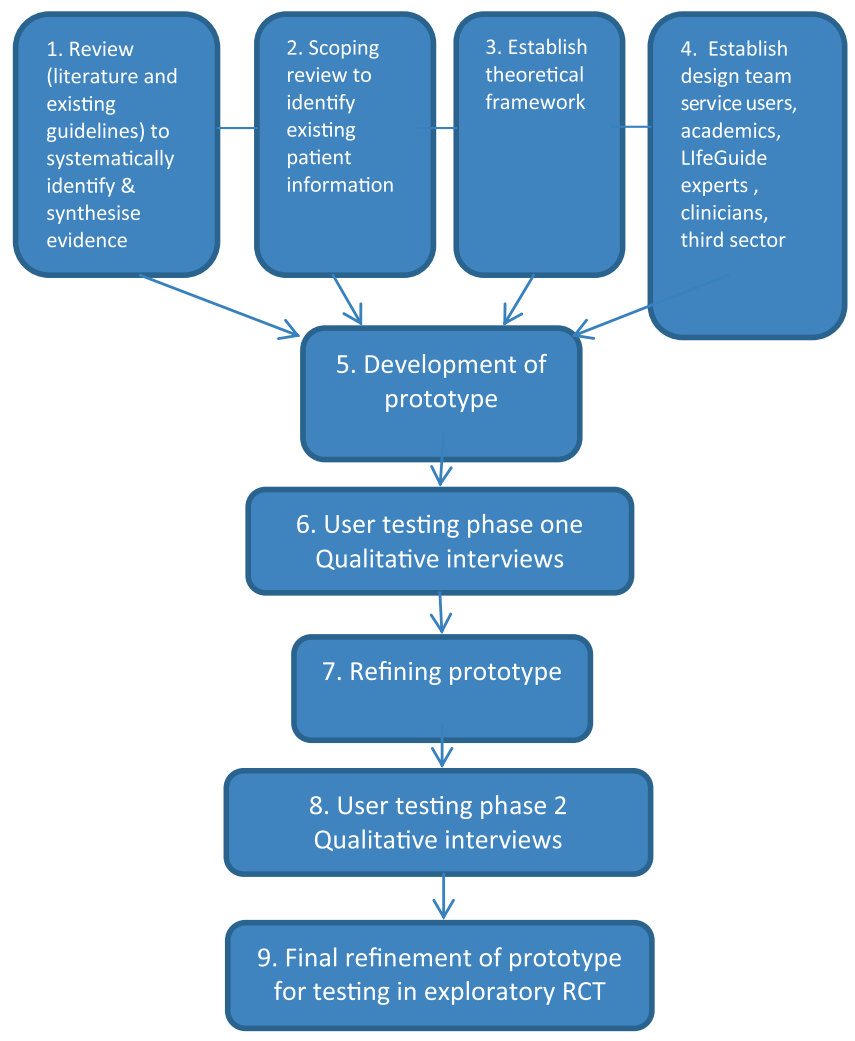

Figure I. Study design

missing, or concepts out of step with the patient experience. Developing a web-based resource to support selfmanagement after cancer treatment was endorsed. In 2012, more specific ideas around RESTORE as a webbased intervention to foster self-efficacy to manage fatigue were discussed, and important contributions were made including the need for the resource to be accessible in terms of literacy, comprehension and level of computer skills required. Attendance was not incentivised, but out-of-pocket expenses (e.g. travel and car parking) were compensated.

\section{Literature review}

Using systematic reviewing techniques [21], a search of published literature and international evidence-based guidelines was conducted to inform the development of RESTORE. The purpose of the literature review was twofold: (i) identify non-pharmacological, evidence-based interventions to manage CRF; and (ii) identify evidencebased strategies for enhancing self-efficacy.

\section{Review of interventions for cancer-related fatigue}

The review of the literature and guidelines identified a number of non-pharmacological interventions to support patients with CRF. Physical activity shows promise [22]; however, optimal frequency, intensity and duration are unknown. There is also growing support for the use of 
CBT, which includes goal setting as a key component [23] and psychosocial support such as stress management and relaxation [24]. The National Comprehensive Cancer Network has published guidance on supporting patients with CRF following treatment [9]. Recommendations include self-monitoring of fatigue levels and energy conservation, as well as CBT, psychosocial intervention and engaging in physical activity.

Commonly, fatigue interventions focus on one component, such as increasing physical activity. These have been criticised for not addressing the multifaceted nature of CRF [25]. Multidimensional, face-to-face interventions have been reported [25], and two published studies report webbased interventions for CRF. These focused on enhancing knowledge and beliefs about CRF using a web-based cancer fatigue class [26] and an individually tailored web-based CRF education programme with the primary outcome of the improvement of CRF [27]. We believe RESTORE to be the first web-based intervention with the primary aim of enhancing self-efficacy to manage CRF specifically and provides evidence-based self-management strategies in addition to education and information provision.

\section{Review of key components related to enhancing self-efficacy}

A number of techniques have been identified that support behaviour change through increasing self-efficacy [28]:

- modelling-learning by observing others;

- coping planning - planning ahead for how to deal with particular situation(s);

- helping people make social comparison, that is, compare themselves with others;

- goal setting-for example, setting small realistic goals to gradually reach a particular level of activity;

- provision of feedback on performance;

- activity planning - for example, timing of activity, pacing and planning rest times;

- diaries.

The most common techniques include providing information on consequences of behaviour, prompting selfmonitoring of behaviour and identifying barriers and/or problem solving [28]. Interventions that use more techniques tended to have larger effect sizes.

\section{Scoping of existing materials for cancer survivors}

Information about CRF was identified from the literature review as an important component of the management of CRF. This search identified a number of resources that were reviewed for quality and applicability to the UK context. The Macmillan Coping with Fatigue booklet [29] was identified as an evidence-based, up-to-date source of information that had undergone expert peer review and consultation with service users and was in widespread use across the UK. The three core elements of this booklet are causes of fatigue, its effects and potential coping strategies. These were adopted as the core elements of information in the RESTORE resource.

\section{Theoretical framework}

Cancer diagnosis and treatment can leave people feeling vulnerable and lacking in confidence, which may act as a barrier to support. Foster and Fenlon [13] have set out a framework for recovery of health and well-being in cancer survivorship, which has self-management and support for selfmanagement as essential components. One of the key elements of the framework is self-efficacy [30], which refers to a person's confidence to achieve particular goals, in this case living with or managing problems associated with CRF. Foster and Fenlon's [13] model predicts that with appropriate support, cancer survivors can self-manage problems related to cancer and its treatment and experience restoration of their well-being. The problem (perception that $\mathrm{CRF}$ is a problem) and environmental factors (the webbased intervention) influence coping appraisal (perception of what is required to manage CRF), and therefore self-efficacy to manage CRF. We therefore postulate that including behavioural techniques that enhance self-efficacy (as per Bandura's theory of self-efficacy) will influence the resolution of the problem, that is, enhance self-efficacy to self-manage the impact of CRF on everyday life. This will in turn influence perception of CRF, that is, it becomes less bothersome. In short, the intervention is designed to increase self-efficacy to manage CRF and therefore reduce the perception of its influence on everyday life (rather than improve CRF per se). Higher self-efficacy has been associated with a greater effort and persistence to cope with obstacles and enhanced well-being [31]. Lower self-efficacy is associated with poorer quality of life and more symptoms, but importantly, self-efficacy can be enhanced by intervention [31]. Bandura [30] proposes four sources of self-efficacy: verbal persuasion, vicarious experiences, performance accomplishment and physiological states; and these were incorporated during intervention development.

\section{Design team}

The development of RESTORE was supported from the outset by a design team (11 members) including academics from a number of institutions and clinicians renowned for their expertise in CRF, people affected by cancer and CRF, a Macmillan cancer information specialist, health psychologists, experts in the development and evaluation of web-based behaviour change interventions and experts in cancer survivorship research.

We also worked closely with a team of intervention development experts with extensive experience in the design and implementation of interventions built using 
LifeGuide open-source software [32]. This software allows non-programmers to create and easily modify web-based interventions, which enabled the team to modify the intervention on the basis of feedback from the design team and user testing. The LifeGuide intervention development experts advised on the architecture, usability, aesthetics and interactivity of the website with particular emphasis on simple display and navigation in order to cater for those with limited computer literacy, and to reduce fatigue when using the website. The initial content provided by the research team was adapted by the LifeGuide intervention development experts to make it more Web appropriate. Structure was added by the use of subheadings, text boxes, bullet points and images, and the content was condensed to fewer paragraphs per page. The language was adapted to be clear and concise, shorter sentences were used and key messages were highlighted in bold.

\section{Development of prototype I}

The research team incorporated the elements identified in steps 1-3; review of empirical literature and existing guidelines, existing patient information (Macmillan's Coping with Fatigue booklet) and theoretical framework, and established a draft content and structure for the intervention.

Duration of intervention: There is limited evidence to guide the duration of interventions. Systematic reviews give some guidance for the length of self-management, self-efficacy and behaviour change interventions [33]. Because of the inconsistency of evidence, a pragmatic decision was made about length on the basis of the amount of information and components deemed essential to impact on self-efficacy. For the purposes of the exploratory trial, RESTORE was divided into five sessions, with the intention that survivors would complete one session per week.

Iterative review and revision process: The research team agreed on initial content based on Macmillan's Coping with Fatigue leaflet, and draft pages were created in LifeGuide's Virtual Research Environment by the LifeGuide intervention development experts. An iterative review process then took place with the design team who scrutinised each session as it was developed. Feedback was managed using the prioritisation model MoSCoW [34]. MoSCoW is a service management tool and allows developers to prioritise the important aspects of a project to ensure the most critical elements are delivered within a specified time frame:

$\mathrm{M}$ - Must have; $\mathrm{S}$ - Should have; $\mathrm{C}$ - Could have;

$\mathrm{W}-$ Would like if time permits

The design team appraised the pages, paying particular attention to accuracy of information, suggested activities and their relevance to the conceptual and theoretical framework, participant burden and readability (i.e. literacy level and visual clarity). The comments received from the design team were tabulated in Excel, and the MoSCoW model was applied. After discussion by the research team, amendments were incorporated (Table 1), and prototype 1 was created.

\section{User testing phase I}

User testing enabled the research team to examine users' interactions with the intervention in order to understand and enhance user engagement. Phase 1 user testing involved think-aloud interviews. Think aloud is an established qualitative method originating in humancomputer interaction research and can be used to establish user satisfaction and acceptability with an intervention [35]. Faulkner [36] suggests that sample sizes of over five increase confidence that usability issues can be identified with more testers equaling greater confidence in results.

Approval from the appropriate university research ethics committee was obtained. Individuals were eligible to participate in the study if they were $\geq 18$ years, had completed primary cancer treatment in the past 5 years and were experiencing CRF. Non-English-speaking participants were excluded as the web-based intervention was developed and tested in English at this stage. Participants were recruited via three routes: local newspapers, members of the User Reference Group consisting of cancer survivors and carers, and a notice placed on the research group's project webpage.

People expressing interest in the study were mailed an information sheet and screening form. The screening

Table I. Modifications based on design team feedback (Must and Should items) and 'think-aloud' interviews

\begin{tabular}{ll}
\hline DT/TA & Modification \\
\hline DT & Add morning and afternoon sections to the fatigue diary \\
DT & Omit yellow text \\
DT & Add speech bubbles and quotation marks to patient stories to \\
DT & emphasise these are personal experiences \\
& Clarify explanation of goal setting task \\
DT & Add an additional introductory page explaining the whole \\
DT & intervention \\
& Clarify terminology \\
DT & Clarify importance of self-monitoring using the fatigue diary and \\
DT & evidence for effectiveness \\
& All sessions reviewed to maintain specificity on fatigue (rather than \\
DT & coping with cancer more generally) \\
TA & Clarification of survivors stories, some were unclear whose \\
TA & perspective they were from \\
& Provide a version of the fatigue diary that could be saved and \\
TA & completed on the computer (rather than print only format) \\
&
\end{tabular}

CRF, cancer-related fatigue; DT, design team; TA, think aloud. 
form included sociodemographics (age and gender), fatigue level using the National Comprehensive Cancer Network [37] 0-10 numeric scale (a score of $\geq 4$ defined as moderate/severe fatigue) and date of cancer diagnosis. Following receipt of a completed screening form, respondents were contacted by telephone to arrange a meeting. Prior to the think-aloud interview, written consent was taken.

Four survivors responded to the advert and participated, three women and one man aged between 43 and 74 years. The average fatigue score for participants was 6 on a 10-point scale (range 3-8). Participants were between 2 and 6 years post-treatment. One participant had a fatigue score of 3 ; however, he perceived his fatigue to negatively impact on daily life and was therefore included in the study.

Participants were presented with draft webpages, following the method used by Yardley et al. [35], one session at a time, and asked to 'think aloud' to say what they thought about each page, focusing on the helpfulness of information provided, relevance of the information, readability, comprehension and ease of navigation. The team encouraged critical feedback to support improvements to the resource. At the end of the audio-recorded think-aloud sessions, participants were asked about their general impressions of the intervention. Thematic analysis of the interview transcripts was manually conducted as 'a method for identifying, analysing and reporting patterns within data' ([38], p. 79). Data were tabulated by theme in Excel, and the MoSCoW matrix was used to prioritise changes made to the prototype.

Although we recruited fewer participants than planned, there were no strongly divergent views, and no new categories emerged from the last interview, so we were confident that the main issues were captured.

\section{Refining prototype}

Major changes to the intervention were not required at this stage, and minor modifications were made (Table 1). Importantly, we identified that it was difficult for participants to relate to stories of cancer survivors as it was unclear what perspective they had on CRF and whether they were speaking as someone currently affected by CRF or reflecting on a past experience. Clarification was therefore incorporated, and pages were re-drafted.

\section{Phase 2 of user testing}

A different set of participants was recruited for phase 2 using the same recruitment routes as phase 1. Additionally, the 'Macmillan Opportunities' webpage-a location where researchers can advertise studies to registered cancer survivors-was used to advertise the study.

The same inclusion criteria were used with the addition that individuals who did not have computer or Internet access were excluded as they were required to have access to these at home.

Upon receipt of a completed screening form, respondents were contacted by a researcher by phone and asked to return a consent form by post. When the consent form was received, participants were sent the link for the intervention via e-mail and asked to trial the intervention for 2 weeks at home. A new session of the intervention was released every 2 days. Time to complete the intervention was truncated as examinations of usability and content were of primary importance with the burden of the intervention in its entirety to be tested in the exploratory randomised controlled trial (RCT). Because of personal reasons (including fatigue levels, personal life, and employment), most participants were only able to do one session per week. A member of the research team prompted participants by email or telephone to log in for their next session.

Participants were asked to complete the intervention (all five sessions when possible) and associated tasks, at home over the course of 2 weeks. As we planned to test RESTORE in an exploratory RCT, we also identified potential outcome measures for participants to test.

Participants agreed to semi-structured, audio-recorded telephone interviews conducted by one experienced qualitative researcher after the completion of every two to three sessions, according to participants' availability. Participants were provided with structured evaluation sheets to keep notes during their use of the intervention, which they could refer to in the telephone interview. The topic guide focused on participants' views of the intervention and any positive or negative views of the sessions. The questions were directed at participants' expectations of the session, their experiences regarding talking to other people and receiving social support prior to the session and any expected changes, benefits or drawbacks after going through the session. The telephone interviews were approximately 30 minutes long.

Ten participants contacted the research team, and seven agreed to participate. Six were women, and the average score for fatigue was 5, on a 10-point scale with a range of 3-7. Two women were still undergoing hormone treatment for breast cancer, and the longest time since treatment was 5 years. The majority $(n=5)$ were diagnosed with breast cancer, and ages ranged from 42 to 70 years. Four participants completed all sessions of the prototype intervention.

As previously performed, participant views were thematically analysed and tabulated in Excel, and the MoSCoW matrix was used to prioritise the suggested modifications (Table 2). Participants also completed the questionnaire measures developed to evaluate the intervention and comment on the level of burden, comprehension and relevance of included items. See Table 2 for amendments.

The interviews revealed that the intervention seemed acceptable to participants. The user testing also 
Table 2. Modifications based on phase 2 interviews to intervention or outcome measures

\begin{tabular}{|c|c|}
\hline I or OM & Modification \\
\hline I & $\begin{array}{l}\text { Re-phrase 'speaking to your doctor' to cover all health professionals; } \\
\text { use of the word 'doctor' was viewed as ambiguous. }\end{array}$ \\
\hline I & $\begin{array}{l}\text { User described experience of feeling like a fraud as he does not have } \\
\text { severe fatigue but moderate fatigue that does have significant } \\
\text { impact on day-to-day life. Added a sentence that people } \\
\text { experience fatigue differently from one another. }\end{array}$ \\
\hline I & $\begin{array}{l}\text { Most patient stories described experiences of people with severe } \\
\text { fatigue; add examples of dealing with less severe fatigue. }\end{array}$ \\
\hline I & $\begin{array}{l}\text { Suggestion of inclusion of how to talk to your GP about fatigue and } \\
\text { communicate its impact on everyday life and the need for } \\
\text { support-we included a link to an information sheet, which } \\
\text { supports this patient/doctor interaction. }\end{array}$ \\
\hline I & $\begin{array}{l}\text { Identified problems with downloading fatigue diary-actioned by } \\
\text { development team. }\end{array}$ \\
\hline I & $\begin{array}{l}\text { Clarify estimated time needed to complete all sessions and that all } \\
\text { sessions do not have to be completed in one go. }\end{array}$ \\
\hline I & $\begin{array}{l}\text { In session 2, asked to select two areas of life for initial goal setting, } \\
\text { participant wanted to select more and suggested adding text to } \\
\text { say can come back to other areas another time. }\end{array}$ \\
\hline 1 & $\begin{array}{l}\text { Identified technical problems with 'take a break' buttons, which were } \\
\text { resolved. }\end{array}$ \\
\hline I & $\begin{array}{l}\text { Re-named the section on physical activity from 'decreased activity' } \\
\text { to 'doing less'. }\end{array}$ \\
\hline I & $\begin{array}{l}\text { Changed the titles of the videos to make them more descriptive, that } \\
\text { is, from video I to 'person talking about coping with fatigue after } \\
\text { prostate cancer'. }\end{array}$ \\
\hline 1 & Identified error in external link, which was corrected. \\
\hline I & Addition of information on talking to children about fatigue. \\
\hline OM & Numbering pages so users can track progress. \\
\hline OM & $\begin{array}{l}\text { Some outcome measures use the word 'illness' when referring } \\
\text { to cancer. Some participants who were several years post- } \\
\text { diagnosis and disease free did not feel this was relevant. An } \\
\text { introductory sentence was added to acknowledge this: 'The word } \\
\text { "illness" relates to your previous cancer diagnosis. If you finished } \\
\text { your cancer treatment some time ago, some of these questions } \\
\text { may not seem as relevant as they did in the past. Please answer } \\
\text { the questions as it applies to you these days.' }\end{array}$ \\
\hline OM & $\begin{array}{l}\text { The questionnaire was felt to be too long-outcome measures } \\
\text { were therefore prioritised and non-essential measures removed. }\end{array}$ \\
\hline 1 & $\begin{array}{l}\text { Link to online forum to enable conversations with other people } \\
\text { affected by cancer. }\end{array}$ \\
\hline
\end{tabular}

I, intervention; OM, outcome measures.

established that the decision to divide the material into five sessions was deemed appropriate with no session being overly burdensome for fatigued individuals. The outcome measures were deemed too long and therefore reduced in length.

\section{Results}

\section{Final intervention}

Final refinements were made as described earlier. Table 3 summarises the RESTORE intervention, which is divided into five sessions and designed to be completed at weekly intervals.
Participants are presented with a welcome page, which outlines the purpose of the intervention. They then have 6 weeks to complete five sessions. Sessions 1 and 2 are compulsory and cover an introduction to $\mathrm{CRF}$, causes and effects, and the concept of goal setting, respectively. Participants can then choose to visit sessions 3-5 depending on what they deem relevant to them. These three sessions are designed to cover most areas of life where CRF may have a negative impact, for example, home and work life, personal relationships and emotional adjustment. Users are encouraged to set weekly SMART goals on the basis of the content of these sessions. When they revisit the intervention, if 1 week has passed since their last visit, they are asked if they have successfully completed their goals, and automated tailored feedback is provided. If goals are successfully completed, participants receive a message of congratulations. If goals are not completed, taking time to reflect on the outcome is suggested, and new revised goals are set. Participants are also reminded of their previous fatigue and confidence levels and given the opportunity to reflect on these. These data are captured by the LifeGuide software for future analysis.

Activities are available throughout RESTORE. These include a fatigue diary that can be downloaded, patient narratives (stories) on experiences of coping with CRF, automated tailored feedback based on goal-related progress and change in fatigue scores. External links to useful resources on trusted websites are available at the end of each session, such as information on financial assistance, the NHS guide to talking therapies and an online forum.

\section{Conclusion}

This paper describes the development of RESTORE; a web-based intervention to enhance self-efficacy to selfmanage CRF after primary cancer treatment. It details the nine steps used to develop the content and appearance of the intervention and processes for involving stakeholders; this provides a model for the development of similar interventions.

For several aspects of the intervention, there was a lack of literature to support evidence-based design, and therefore, as reported by Michie et al. [19], some decisions about the intervention were based on the judgement of the design team, such as the length of the intervention. However, this has been made transparent, and any areas of uncertainty were addressed through user testing. The intervention is multifaceted, but by detailing the components of the intervention, which are based on theory and empirical evidence, the mechanisms of change integrated into the content are made clear, reducing the 'black box' criticism of complex interventions, that is, being able to state the active ingredients within the intervention and how they work [18]. 
Table 3. Intervention content

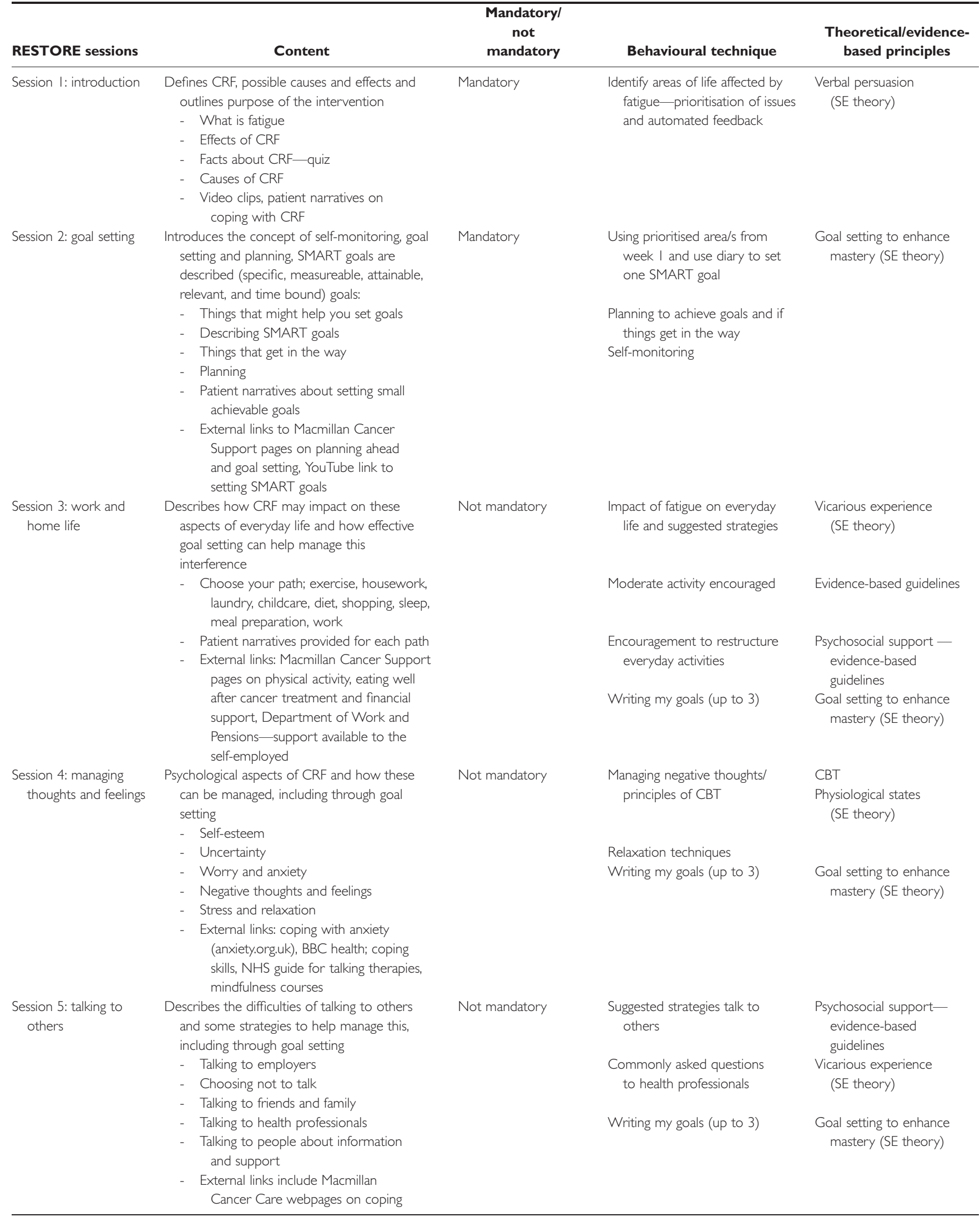


Table 3. (Continued)

\begin{tabular}{|c|c|c|c|c|}
\hline RESTORE sessions & Content & $\begin{array}{l}\text { Mandatoryl } \\
\text { not } \\
\text { mandatory }\end{array}$ & Behavioural technique & $\begin{array}{l}\text { Theoretical/evidence- } \\
\text { based principles }\end{array}$ \\
\hline & $\begin{array}{l}\text { emotionally, relationship communication, } \\
\text { talking to children, and online forum, } \\
\text { healthtalkonline to hear about others' } \\
\text { experiences, link to a information } \\
\text { on enhancing communication } \\
\text { with GPs }\end{array}$ & & & \\
\hline \multicolumn{5}{|c|}{ Activities suggested throughout } \\
\hline $\begin{array}{l}\text { Provision of } \\
\text { information }\end{array}$ & $\begin{array}{l}\text { Information about fatigue and suggested } \\
\text { strategies for management based on } \\
\text { Macmillan 'Coping with Fatigue' booklet }\end{array}$ & Mandatory in session I & & $\begin{array}{l}\text { Evidence-based guidelines/ } \\
\text { vicarious experience } \\
\text { (SE theory) }\end{array}$ \\
\hline $\begin{array}{l}\text { Diary and } \\
\text { self-monitoring }\end{array}$ & $\begin{array}{l}\text { Fatigue diary to enable participants to reflect } \\
\text { on how they feel physically and emotionally, } \\
\text { plan activities around patterns of fatigue and } \\
\text { assess if their experience of fatigue has } \\
\text { changed } \\
\text { Monitoring fatigue each time a participant logs } \\
\text { in to RESTORE }\end{array}$ & Not mandatory & & $\begin{array}{l}\text { Physiological states } \\
\text { (SE theory) }\end{array}$ \\
\hline Patient stories & $\begin{array}{l}\text { Extracts from people affected by cancer and } \\
\text { who have experienced fatigue; sharing } \\
\text { their experiences and methods of self- } \\
\text { management, written text and video clips }\end{array}$ & Not mandatory & & $\begin{array}{l}\text { Vicarious experience } \\
\text { (SE theory) }\end{array}$ \\
\hline Feedback & $\begin{array}{l}\text { Tailored feedback on success of goal setting, } \\
\text { planning and fatigue level }\end{array}$ & Mandatory & & $\begin{array}{l}\text { Verbal persuasion } \\
\text { (SE theory) }\end{array}$ \\
\hline Web links & Web links to useful resources & Not mandatory & & $\begin{array}{l}\text { Vicarious experience } \\
\text { (SE theory)/information- } \\
\text { evidence-based guidelines }\end{array}$ \\
\hline Take a break buttons & $\begin{array}{l}\text { Provided throughout to allow participants to } \\
\text { rest during sessions if required }\end{array}$ & Not mandatory & & N/A \\
\hline
\end{tabular}

CBT, cognitive behaviour therapy; CRF, cancer-related fatigue; SE, self-efficacy.

Of critical importance was the co-creation of the intervention with the design team and service users. This intervention was targeted at people who were already fatigued, which required a significant amount of user involvement to ensure that the intervention was acceptable and met the needs of people who may not be able to spend long periods engaged with it.

Although participants did not find the intervention sessions per se overly burdensome, additional tasks of feeding back to the research team and participating in telephone interview were too great a workload for some, and thus, not all RESTORE sessions were completed. Gauging the acceptability of workload for those involved in user testing should be considered in the development of future interventions.
The importance of the involvement of service users in the design and conduct of research is now well recognised [39]. The science of complex intervention development is still in the early stages of development, and few studies have fully described this phase [19]. Previous literature has described how stakeholder involvement can radically change the approach of researchers [40] and significantly contribute to the development of interventions [41], demonstrating the importance of user involvement to enhance the acceptability of interventions to end users. Different ways of involving stakeholders have been reported, including user involvement in consensus exercises to identify intervention components [42] and intervention points [43]. This paper demonstrates a comprehensive approach to stakeholder involvement 
illustrating that they can be involved in every stage of development.

The use of LifeGuide open-source software and the involvement of LifeGuide intervention development experts made iterative development of the intervention a relatively straightforward process, without the need for programmers. However, the process of incorporating feedback from user testing and the design team and modifying the prototype accordingly was time consuming, and future studies should allow for this in their development time scale.

The RESTORE intervention will be tested in a proof of concept exploratory RCT [44]. A process evaluation embedded in the feasibility trial design will seek to further understand the mechanisms of change and the acceptability of the intervention to fatigued cancer survivors.

In summary, this paper describes the development of a novel, evidence-based, theory-driven, web-based, selfmanagement intervention to support cancer survivors living with CRF and is a potential model for others, advancing the field of supported self-management interventions.

\section{Acknowledgements}

This study was funded by Macmillan Cancer Support. We thank the participants, members of the Trial Steering Committee and members of the Macmillan Survivorship Research Group who supported this study.

\section{References}

1. World Cancer Research Fund/American Institute for Cancer Research. Food, Nutrition, Physical Activity, and the Prevention of Cancer: A Global Perspective. Washington DC, 2007.

2. Hewitt M, Rowland JH, Yancik R. Cancer survivors in the United States: age, health, and disability. J Gerontol A Biol Sci Med Sci 2003;58(1):M82.

3. Foster C et al. Psychosocial implications of living 5 years or more following a cancer diagnosis: a systematic review of the research evidence. Eur J Cancer Care (Engl) 2009;18(3):223-247.

4. Armes J et al. Patients' supportive care needs beyond the end of cancer treatment: a prospective, longitudinal survey. J Clin Oncol 2009;27(36):6172.

5. NCSI. Living with and beyond cancer: taking action to improve outcomes https://www.gov. uk/government/publications/living-with-andbeyond-cancer-taking-action-to-improve-outcomes Accessed 12/6/2013. 2013.

6. Bower JE et al. Fatigue in breast cancer survivors: occurrence, correlates, and impact on quality of life. J Clin Oncol 2000; 18(4):743.

7. Foster $\mathrm{C}$ et al. Cancer survivors' self-efficacy to self-manage in the year following primary treatment. J Cancer Surviv 2014. DOI: 10.1007/s11764-014-0384-0

8. Servaes $\mathrm{P}$ et al. Fatigue after breast cancer and in chronic fatigue syndrome: similarities and differences. J Psychosom Res 2002;52(6): 453-459.

9. National Comprehensive Cancer Network. NCCN clinical practice guidelines in oncology-cancer-related fatigue. National Comprehensive Cancer Network, Version 1.2013: Fort Washington PA, 2014.

10. Hoffman A et al. Testing a theoretical model of perceived self-efficacy for cancerrelated fatigue self-management and optimal physical functional status. Nurs Res 2009; 58(1):32-41.
11. Porter LS et al. Self-efficacy for managing pain, symptoms, and function in patients with lung cancer and their informal caregivers: associations with symptoms and distress. Pain 2008;137(2):306-315.

12. Phillips SM, McAuley E. Physical activity and fatigue in breast cancer survivors: a panel model examining the role of self-efficacy and depression. Cancer Epidemiol Biomarkers Prev 2013;22(5):773-781.

13. Foster C, Fenlon D. Recovery and selfmanagement support following primary cancer treatment. Br J Cancer 2011;105(S1): S21-S28.

14. Murray E. web-based interventions for behavior change and self-management: potential, pitfalls, and progress. Med 20 2012;1(2):e3.

15. Chou W-yS et al. Health-related Internet use among cancer survivors: data from the Health Information National Trends Survey, 2003-2008. J Cancer Surviv 2011;5(3): 263-270.

16. Randall C. e-Society, Social Trends 41 . Office for National Statistics, 2010.

17. Yardley L, Nyman SR. Internet provision of tailored advice on falls prevention activities for older people: a randomized controlled evaluation. Health Promot Int 2007; 22(2): 122

18. Medical Research Council. Developing and Evaluating Complex Interventions: New Guidance. MRC: London, 2008.

19. Michie S et al. Development of StopAdvisor: a theory-based interactive internet-based smoking cessation intervention. Transl Behav Med 2012;2(3):263-275.

20. Yardley L et al. Integrating user perspectives into the development of a web-based weight management intervention. Clinical Obesity 2013;2(5-6):132-141.

21. Centre for Reviews and Dissemination. Systematic Reviews: CRD's Guidance for Undertaking Systematic Reviews in Health Care. CRD: University of York, 2009.

22. Cramp F, Byron-Daniel J. Exercise for the management of cancer-related fatigue in adults. Cochrane Database Syst Rev 2012;(11)

23. Gielissen MF, Verhagen CA, Bleijenberg G. Cognitive behaviour therapy for fatigued cancer survivors: long-term follow-up. Br J Cancer 2007;97(5):612-618.

24. Reif $\mathrm{K}$ et al. [What does really help against cancer-related fatigue? An overview of systematic reviews]. Was hilft wirklich bei tumorbedingter Fatigue? Ein Überblick über systematische Übersichtsarbeiten. Pflege 2012;25(6):439-457.

25. Stuhldreher N et al. Development and evaluation of a cancer-related fatigue patient education program: protocol of a randomized controlled trial. BMC Nurs 2008;7:12.

26. Smith $\mathrm{T}$ et al. Theory-based evaluation of an online cancer fatigue class. J Cancer Educ 2010;25(3):422-430.

27. Yun $\mathrm{YH}$ et al. Web-based tailored education program for disease-free cancer survivors with cancer-related fatigue: a randomized controlled trial. J Clin Oncol 2012; 30(12):1296-1303.

28. Abraham C, Michie S A taxonomy of behavior change techniques used in interventions. Health Psychol 2008;27(3):379-387.

29. Macmillan Cancer Support. Coping with Fatigue (5th edn). MAC11664. 2011. http:// be.macmillan.org.uk/be/p-284-coping-withfatigue.aspx.

30. Bandura A. Social Foundations of Thought and Action: A Cognitive Social Theory. Prentice Hall: Englewood Cliffs, New York, 1986.

31. Lorig KR et al. Chronic disease selfmanagement program-2-year health status and health care utilization outcomes. Med Care 2001;39(11):1217-1223.

32. Hare $\mathrm{J}$ et al. LifeGuide: a platform for performing web-based behavioural interventions. WebSci09: Society On-Line, 2009.

33. Cugelman B, Thelwall M, Dawes P. Online interventions for social marketing health behavior change campaigns: a meta-analysis of psychological architectures and adherence factors. J Med Internet Res 2011; 13(1):e17. 
34. Kuhn J. Decrypting the MoSCoW analysis (DITY weekly newsletter). itSM solutions, 2009. 5: p. 44.

35. Yardley L et al. Understanding reactions to an internet-delivered health-care intervention: accommodating user preferences for information provision. BMC Med Inform Decis Mak 2010;10(1):52.

36. Faulkner L Beyond the five-user assumption: benefits of increased sample sizes in usability testing. Behav Res Methods Instrum Comput 2003;35(3):379-383.

37. Network NCC. NCCN Clinical Practice Guidelines in Oncology: Cancer-Related Fatigue. Version 1.2012. National Comprehensive Cancer Network: Fort Washington, $\mathrm{Pa}, 2011$.
38. Braun V, Clarke V. Using thematic analysis in psychology. Qual Res Psychol 2006;3(2): 77-101.

39. Oliver S, Clarke-Jones L, Rees R. Involving consumers in research and development agenda setting for the NHS: developing an evidence-based approach, in Health Technology Assessment Reports. 2004.

40. Owens $\mathrm{C}$ et al. Involving service users in intervention design: a participatory approach to developing a text-messaging intervention to reduce repetition of self-harm. Health Expect 2011;14(3):285-295.

41. Lowes L et al. Involving lay and professional stakeholders in the development of a research intervention for the DEPICTED study. Health Expect 2011;14(3):250-260.
42. Lovell $\mathrm{K}$ et al. Developing guided self-help for depression using the Medical Research Council complex interventions framework: a description of the modelling phase and results of an exploratory randomised controlled trial. BMC Psychiatry 2008;8(1):1-19.

43. Hardeman W et al. A causal modelling approach to the development of theorybased behaviour change programmes for trial evaluation. Health Educ Res 2005;20(6): 676-687.

44. Grimmett $\mathrm{C}$ et al. RESTORE: an exploratory trial of an online intervention to enhance self-efficacy to manage problems associated with cancer-related fatigue following primary cancer treatment: study protocol for a randomized controlled trial. Trials 2013;14:184. 